\title{
Ancestral SARS-CoV-2-specific T cells cross-recognize the Omicron variant
}

\author{
Yu Gao,11, Curtis Cai ${ }^{1,11}$, Alba Grifoni ${ }^{2}$, Thomas R. Müller ${ }^{1}$, Julia NiessI ${ }^{1}$, \\ Anna Olofsson ${ }^{3}$, Marion Humbert $\mathbb{B}^{3}{ }^{3}$, Lotta Hansson ${ }^{4,5}$, Anders Österborg ${ }^{4,5}$, Peter Bergman ${ }^{3,6}$, \\ Puran Chen', Annika Olsson ${ }^{6}$, Johan K. Sandberg ${ }^{\circledR 1}{ }^{1}$, Daniela Weiskopf ${ }^{2}$, David A. Price ${ }^{7,8}$, \\ Hans-Gustaf Ljunggren', Annika C. Karlsson $\mathbb{1}^{3}{ }^{3}$, Alessandro Sette $\mathbb{1}^{2,9}$, Soo Aleman ${ }^{6,10}$ and \\ Marcus Buggert $\mathbb{1}^{1}$ 瓜
}

\begin{abstract}
The emergence of the severe acute respiratory syndrome coronavirus 2 (SARS-CoV-2) Omicron (B.1.1.529) variant of concern (VOC) has destabilized global efforts to control the impact of coronavirus disease 2019 (COVID-19). Recent data have suggested that B.1.1.529 can readily infect people with naturally acquired or vaccine-induced immunity, facilitated in some cases by viral escape from antibodies that neutralize ancestral SARS-CoV-2. However, severe disease appears to be relatively uncommon in such individuals, highlighting a potential role for other components of the adaptive immune system. We report here that SARS-CoV-2 spike-specific CD4 ${ }^{+}$ and $\mathrm{CDB}^{+} \mathrm{T}$ cells induced by prior infection or BNT162 b2 vaccination provide extensive immune coverage against B.1.1.529. The median relative frequencies of SARS-CoV-2 spike-specific CD4 $^{+} \mathrm{T}$ cells that cross-recognized B.1.1.529 in previously infected or BNT162b2-vaccinated individuals were $84 \%$ and $91 \%$, respectively, and the corresponding median relative frequencies for SARS-CoV-2 spike-specific CD8 ${ }^{+} \mathbf{T}$ cells were $70 \%$ and $92 \%$, respectively. Pairwise comparisons across groups further revealed that SARS-CoV-2 spike-reactive CD4 ${ }^{+}$ and $\mathrm{CDB}^{+} \mathbf{T}$ cells were functionally and phenotypically similar in response to the ancestral strain or B.1.1.529. Collectively, our data indicate that established SARS-CoV-2 spike-specific $\mathrm{CD4}^{+}$and $\mathrm{CD8}^{+} \mathrm{T}$ cell responses, especially after BNT162 b2 vaccination, remain largely intact against B.1.1.529.
\end{abstract}

Natural infection with SARS-CoV-2 and vaccination with messenger RNA (mRNA) constructs encoding the viral spike protein typically generate effective immunity against COVID19. However, the current pandemic has been fueled by the continual emergence of VOCs, such as Omicron (B.1.1.529). Recent data indicate that B.1.1.529 is more transmissible than previous VOCs ${ }^{1}$. This phenotype can be explained by key mutations in the receptor-binding domain, which confer enhanced affinity for the ACE2 receptor $^{2}$. Another major concern is that B.1.1.529 harbors a large number of additional mutations in the spike protein that could feasibly subvert immune recognition (https://www.ecdc. europa.eu/sites/default/files/documents/threat-assessment-covid -19-emergence-sars-cov-2-variant-omicron-december-2021.pdf). In line with this possibility, emerging reports have shown that neutralizing antibodies elicited against the ancestral Wuhan reference strain, in the context of either infection or vaccination, are less able to combat B.1.1.529 (refs. ${ }^{2,3}$ ). These observations likely align with the propensity of B.1.1.529 to cause breakthrough infections $s^{4,5}$.

Preliminary data suggest that breakthrough infections with B.1.1.529 are associated with a lower risk of hospitalization and/ or severe illness compared with the Delta VOC (B.1.617.2) (refs. $\left.{ }^{6,7}\right)$. One possible inference from these clinical observations is that additional immune mechanisms beyond antibody production attenuate the course of infection with B.1.1.529. Previous studies have demonstrated that robust $\mathrm{CD}^{+}$and $\mathrm{CD} 8^{+} \mathrm{T}$ cell responses are induced following SARS-CoV-2 infection or vaccination ${ }^{8-14}$. Several lines of evidence further suggest that $\mathrm{CD}^{+}$and $\mathrm{CD}^{+}$ $\mathrm{T}$ cell responses can modulate disease severity in humans and suppress viral replication in animal models $s^{15-18}$. However, it has remained unclear to what extent ancestral SARS-CoV-2-specific $\mathrm{CD}^{+}$and $\mathrm{CD}^{+} \mathrm{T}$ cells cross-recognize B.1.1.529, especially given the unprecedented number of mutations in the spike protein, which likely shift the antigenic landscape more profoundly in relation to antecedent VOCs ${ }^{19}$.

To address this question, we collected peripheral blood mononuclear cells (PBMCs) from vaccinated individuals 6 months after a second dose of the Pfizer/BioNTech mRNA BNT162b2 formulation (median age, 53 years; $n=23$ females and 17 males), individuals in the convalescent phase 9 months after mild (median age, 54 years; $n=8$ females and 18 males) or severe COVID-19 (median age, 58 years; $n=3$ females and 19 males) and seronegative individuals (unclassified demographics, total $n=48$ ) (Supplementary Table 1). Cells were stimulated in parallel with overlapping peptide pools spanning the entire spike protein sequences of the Wuhan reference strain (wild-type) or B.1.1.529. Activation-induced marker assays were used to quantify spike-specific $\mathrm{CD}^{+} \mathrm{T}$ cell responses via the upregulation of CD69 and CD40L (CD154) and spike-specific CD8 ${ }^{+}$ $\mathrm{T}$ cell responses via the upregulation of CD69 and 4-1BB (CD137) (Extended Data Fig. 1a).

'Department of Medicine Huddinge, Center for Infectious Medicine, Karolinska Institutet, Stockholm, Sweden. ${ }^{2}$ Center for Infectious Disease and Vaccine Research, La Jolla Institute for Immunology, La Jolla, CA, USA. ${ }^{3}$ Division of Clinical Microbiology, Department of Laboratory Medicine, Karolinska Institutet, Stockholm, Sweden. ${ }^{4}$ Department of Hematology, Karolinska University Hospital, Stockholm, Sweden. ${ }^{5}$ Department of Oncology-Pathology, Karolinska Institutet, Stockholm, Sweden. ${ }^{6}$ Department of Infectious Diseases, Karolinska University Hospital, Stockholm, Sweden. ${ }^{7}$ Division of Infection and Immunity, Cardiff University School of Medicine, University Hospital of Wales, Cardiff, UK. ${ }^{8}$ Systems Immunity Research Institute, Cardiff University School of Medicine, University Hospital of Wales, Cardiff, UK. 'Division of Infectious Diseases and Global Public Health, Department of Medicine, University of California, San Diego, La Jolla, CA, USA. ${ }^{10}$ Infectious Diseases and Dermatology Unit, Department of Medicine Huddinge, Karolinska

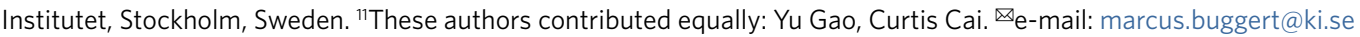




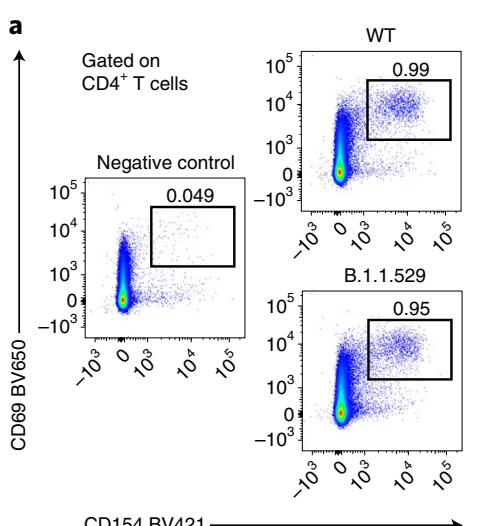

b

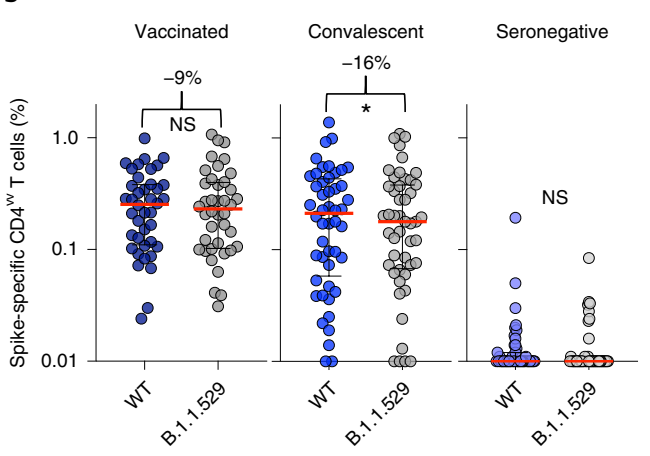

c

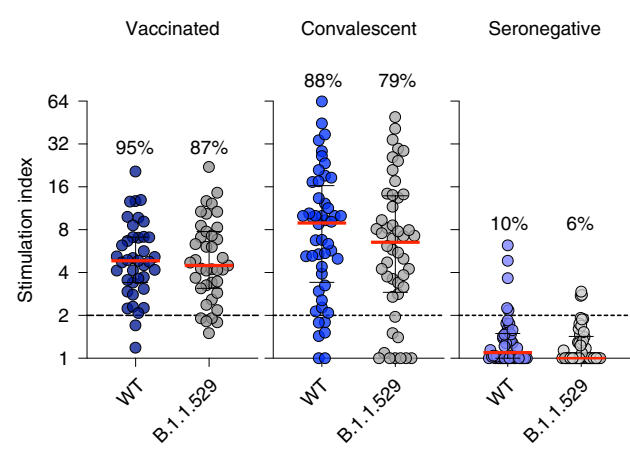

d
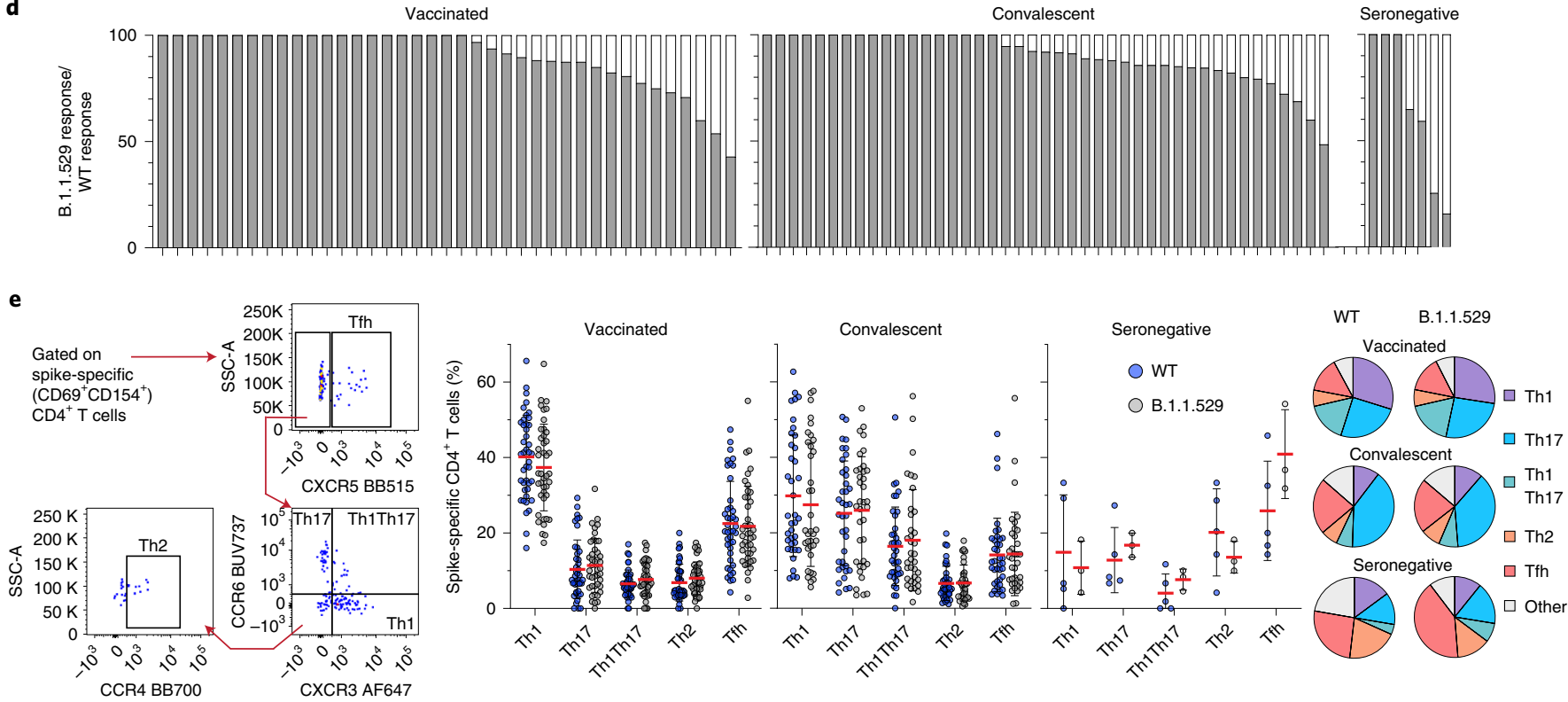

f
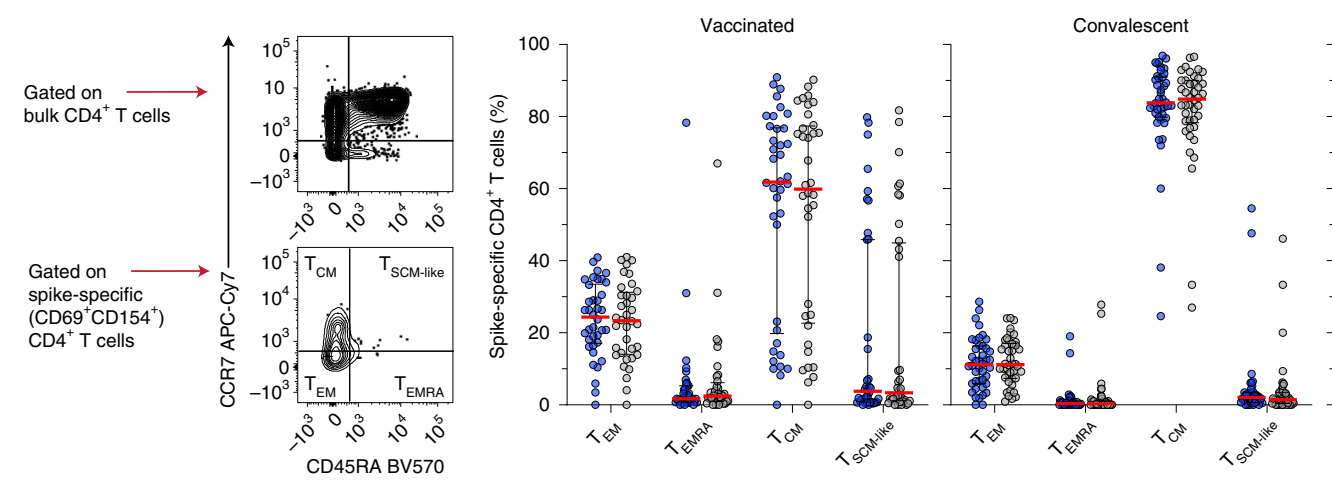

Seronegative

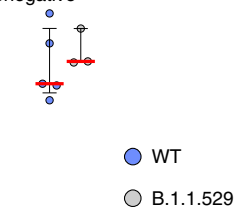

g

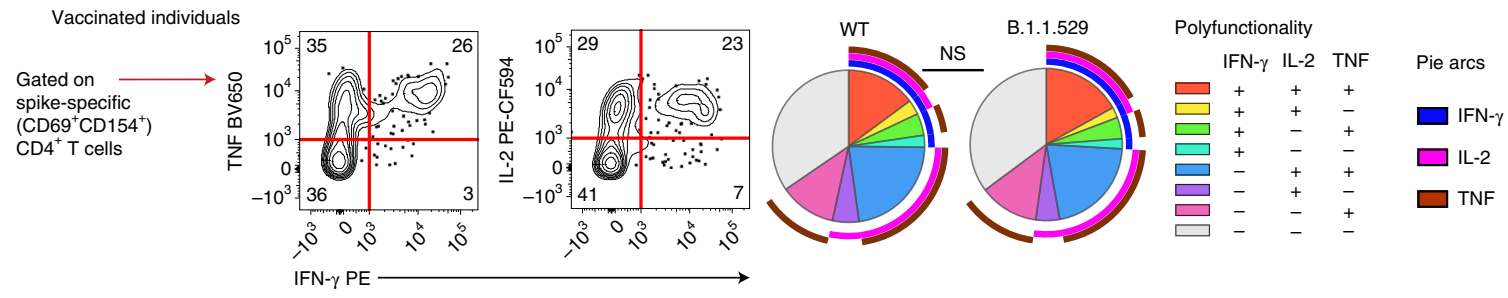

The overall magnitude of the SARS-CoV-2 spike-specific CD4 ${ }^{+}$ $\mathrm{T}$ cell response against B.1.1.529 showed a median reduction of $9 \%$ in BNT162b2-vaccinated individuals and a median reduction of $16 \%$ in convalescent individuals relative to the wild-type response
(Fig. 1a,b). The corresponding response frequencies, defined using a threshold stimulation index, were also slightly lower for B.1.1.529 (Fig. 1c). Pairwise comparisons further revealed maximum reductions in magnitude of 58\% among BNT162b2-vaccinated individu- 
Fig. 1 | Cross-reactive CD4+ T cell responses against B.1.1.529. a, Representative flow cytometry plots showing spike-specific CD4+ $T$ cell responses $\left(\mathrm{CD} 69^{+} \mathrm{CD} 154^{+}\right)$to peptide pools representing wild-type SARS-CoV-2 (WT) or B.1.1.529. b, Frequencies of all spike-specific CD4 ${ }^{+}$T cells in BNT162b2-vaccinated, convalescent and seronegative individuals. Numbers indicate median reduction in the frequency of detected responses. Comparisons used two-sided Wilcoxon signed rank tests. ${ }^{\star} P=0.012$. c, Stimulation indices calculated as fold change in frequency relative to the negative control. Numbers indicate the percentage of individuals with a detectable response. $\mathbf{d}$, Cross-reactive responses depicted on an individual basis as percentage B.1.1.529/wild-type. e, Helper polarization of spike-specific CD4 ${ }^{+} \mathrm{T}$ cells with representative gating and dot plots showing the distribution of subsets across individuals with detectable responses. Pie charts show the mean frequency of each subset across all individuals in each group. $\mathbf{f}$, Canonical memory differentiation profiles of spike-specific CD4+ $\mathrm{T}$ cells with representative gating and dot plots showing the distribution of subsets across individuals with detectable responses. $\mathbf{g}$, Functional profiles of spike-specific CD4+ T cell responses in BNT162b2-vaccinated individuals with representative gating and pie charts showing the mean frequency for each combination. Polyfunctional responses were compared using a permutation test. Data in dot plots are shown as median \pm interquartile range. Each dot represents one donor.

als who were vaccinated, $56 \%$ among convalescent individuals and $75 \%$ among individuals who were seronegative for SARS-CoV-2 spike-specific $\mathrm{CD}^{+} \mathrm{T}$ cell responses against B.1.1.529 versus wild-type (Fig. 1d). These results were validated using independently synthesized peptide pools spanning each spike protein (Extended Data Fig. 1b).

To extend these findings, we investigated the phenotypic characteristics of SARS-CoV-2 spike-specific $\mathrm{CD}^{+} \mathrm{T}$ cells that cross-recognized B.1.1.529, with a particular focus on markers of T helper polarization (CCR4, CCR6, CXCR3 and CXCR5) and memory differentiation (CCR7 and CD45RA). No significant differences in $\mathrm{T}$ helper polarization were detected across intragroup comparisons of SARS-CoV-2 spike-specific CD4 ${ }^{+} \mathrm{T}$ cell responses against B.1.1.529 versus wild-type (Fig. 1e). Central memory $\mathrm{T}$ cells predominated among SARS-CoV-2 spike-specific $\mathrm{CD}^{+}$ $\mathrm{T}$ cells in BNT162b2-vaccinated, convalescent and seronegative individuals, but again, no significant differences in subset composition were detected across intragroup comparisons of SARS-CoV-2 spike-specific $\mathrm{CD}^{+}{ }^{+} \mathrm{T}$ cell responses against B.1.1.529 versus wild-type (Fig. 1f). We also assessed the functionality of SARS-CoV-2 spike-specific CD4 ${ }^{+} \mathrm{T}$ cells in BNT162b2-vaccinated individuals, measuring the intracellular expression of interferon- $\gamma$, tumor necrosis factor and interleukin-2 alongside CD69 and CD154. No significant differences in the ability of SARS-CoV-2 spike-specific $\mathrm{CD} 4^{+} \mathrm{T}$ cells to deploy multiple functions were apparent in response to stimulation with peptides representing B.1.1.529 versus wild-type (Fig. 1g).

The overall magnitude of the SARS-CoV-2 spike-specific $\mathrm{CD}^{+}$ $\mathrm{T}$ cell response against B.1.1.529 showed a median reduction of $8 \%$ in BNT162b2-vaccinated individuals and a median reduction of $30 \%$ in convalescent individuals relative to the wild-type response (Fig. 2a,b). These differences were mirrored in the corresponding response frequencies, defined using a threshold stimulation index (Fig. 2c). Pairwise comparisons further revealed maximum reductions in magnitude of $55 \%$ among BNT162b2-vaccinated individuals, $63 \%$ among convalescent individuals and $60 \%$ among seronegative individuals for SARS-CoV-2 spike-specific $\mathrm{CD}^{+} \mathrm{T}$ cell responses against B.1.1.529 versus wild-type (Fig. 2d). These results were again validated using independently synthesized peptide pools spanning each spike protein (Extended Data Fig. 1c).

In further experiments, we investigated the phenotypic characteristics of SARS-CoV-2 spike-specific $\mathrm{CD}^{+} \mathrm{T}$ cells that cross-recognized B.1.1.529, focusing on classic markers of memory differentiation (CCR7 and CD45RA). Late effector memory T cells predominated among SARS-CoV-2 spike-specific $\mathrm{CD}^{+} \mathrm{T}$ cells in BNT162b2-vaccinated, convalescent and seronegative individuals, but no significant differences in subset composition were detected across intragroup comparisons of SARS-CoV-2 spike-specific CD8 ${ }^{+}$ $\mathrm{T}$ cell responses against B.1.1.529 versus wild-type (Fig. 2e). We also assessed the functionality of SARS-CoV-2 spike-specific CD8 ${ }^{+}$ T cells in BNT162b2-vaccinated individuals, measuring the intracellular expression of granzyme B, interferon- $\gamma$, tumor necrosis factor and interleukin-2 alongside CD69 and CD137. Akin to the corresponding analyses of SARS-CoV-2 spike-specific CD4 ${ }^{+} \mathrm{T}$ cells, no significant differences in the ability of SARS-CoV-2 spike-specific $\mathrm{CD} 8^{+} \mathrm{T}$ cells to deploy multiple functions were apparent in response to stimulation with peptides representing B.1.1.529 versus wild-type (Fig. 2f).

Finally, we merged the SARS-CoV-2 spike-specific $\mathrm{CD}^{+}$and $\mathrm{CD}^{+} \mathrm{T}$ cell data by group, aiming to evaluate cross-recognition en masse. The overall magnitude of the combined SARS-CoV-2 spike-specific $\mathrm{CD}^{+}$and $\mathrm{CD}^{+} \mathrm{T}$ cell response against B.1.1.529 was significantly lower in convalescent individuals, but not in BNT162b2-vaccinated individuals, relative to the wild-type response (Extended Data Fig. 1d). Although potentially reflecting differences in the chronology and/or context of antigen exposure, these results suggest that ancestral SARS-CoV-2 spike-specific CD4 ${ }^{+}$and $\mathrm{CD}^{+}$ $\mathrm{T}$ cells elicited by natural infection provide comprehensive but relatively incomplete coverage against B.1.1.529.

The current global pandemic has been destabilized by the recent emergence of B.1.1.529, which continues to spread rapidly and supersede other VOCs. Our collective data indicate that SARS-CoV-2 spike-specific $\mathrm{CD}^{+}$and $\mathrm{CD}^{+}{ }^{+} \mathrm{T}$ cells elicited by BNT162b2 vaccination or prior infection remain largely intact against B.1.1.529. Alongside intrinsic viral factors, such as altered tropism and decreased replication in the lower respiratory tract ${ }^{20}$, such heterologous immune reactivity may explain why severe disease appears to be relatively uncommon after infection with this particular VOC. Moreover, the degree of cross-reactivity varied to some extent among individuals, most likely as a consequence of genetically encoded differences in antigen presentation, which could further modulate clinical outcomes associated with B.1.1.529. It should be noted that we did not formally assess cytotoxic functions beyond the expression of granzyme $\mathrm{B}$ and that our evaluations were confined to peripheral blood samples, which do not necessarily reflect the entirety of the cellular immune response against SARS-CoV-2 (ref. ${ }^{21}$ ). In addition, we found that SARS-CoV-2 spike-specific $\mathrm{CD}^{+}$and $\mathrm{CD}^{+} \mathrm{T}$ cells cross-recognized B.1.1.529 less comprehensively in convalescent versus BNT162b2-vaccinated individuals, suggesting that booster immunization may provide benefits that extend beyond the induction of broadly neutralizing antibodies to enhance natural protection against recurrent episodes of COVID-19 (ref. ${ }^{2}$ ).

\section{Online content}

Any methods, additional references, Nature Research reporting summaries, source data, extended data, supplementary information, acknowledgements, peer review information; details of author contributions and competing interests; and statements of data and code availability are available at https://doi.org/10.1038/ s41591-022-01700-x.

Received: 30 December 2021; Accepted: 14 January 2022;

Published online: 14 January 2022 


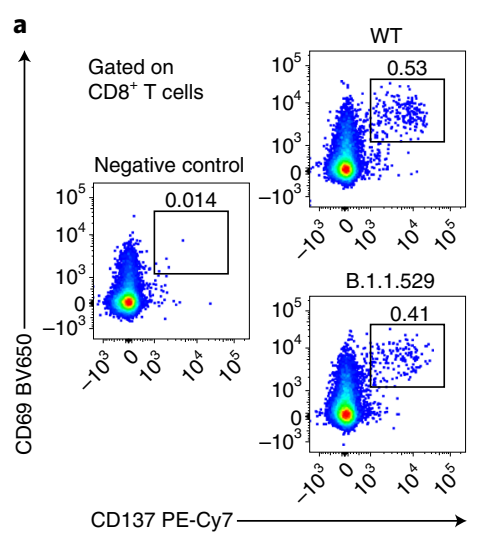

b

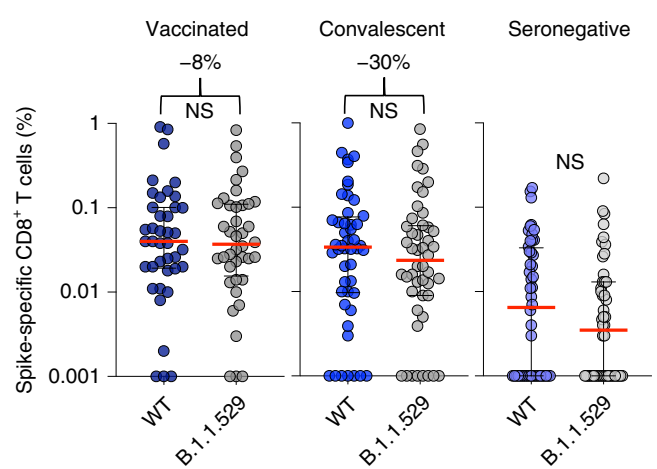

c

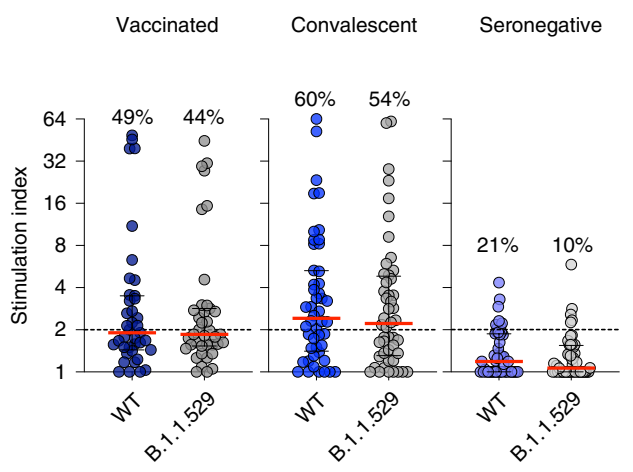

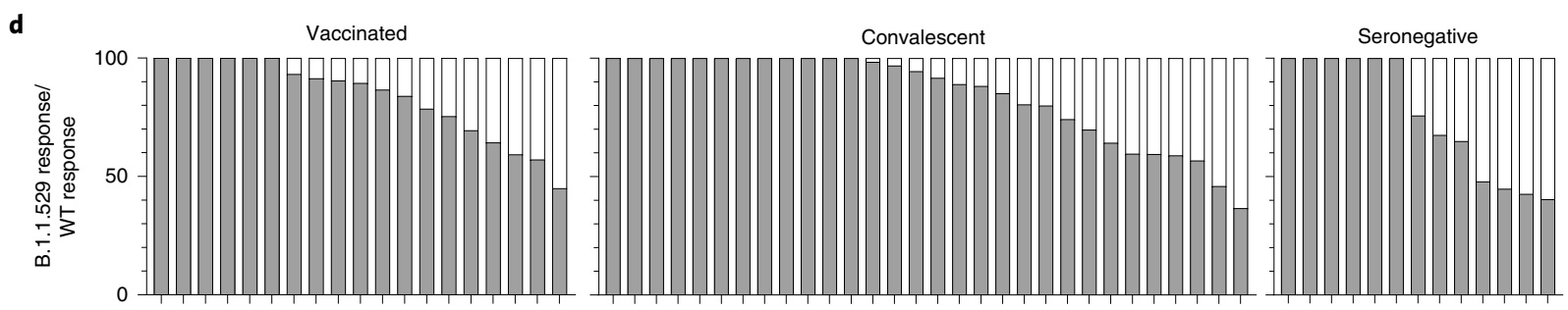
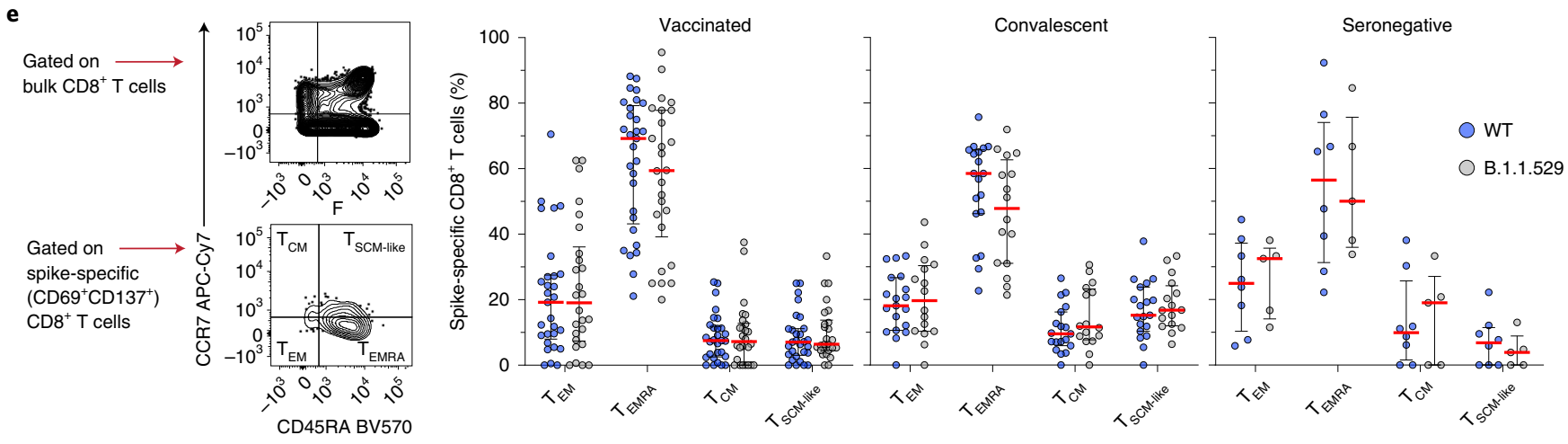

$\mathbf{f}$

Vaccinated individuals

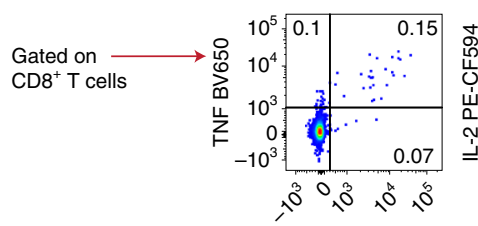

IFN- $\gamma$ PE
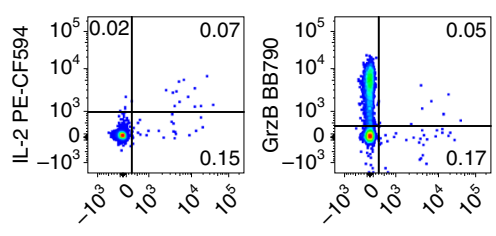
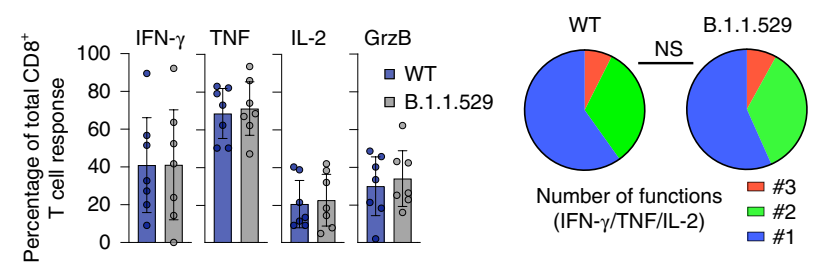

Fig. 2 | Cross-reactive CD8 ${ }^{+} \mathrm{T}$ cell responses against B.1.1.529. a, Representative flow cytometry plots showing spike-specific $C D 8^{+} T$ cell responses $\left(\mathrm{CD} 69^{+} \mathrm{CD} 137^{+}\right)$to peptide pools representing wild-type SARS-CoV-2 (WT) or B.1.1.529. b, Frequencies of all spike-specific CD8 ${ }^{+} \mathrm{T}$ cells in BNT162b2-vaccinated, convalescent and seronegative individuals. Numbers indicate median reduction in the frequency of detected responses. Comparisons used two-sided Wilcoxon signed rank tests. c, Stimulation indices calculated as fold change in frequency relative to the negative control. Numbers indicate the percentage of individuals with a detectable response. $\mathbf{d}$, Cross-reactive responses depicted on an individual basis as percentage B.1.1.529/wild-type. e, Canonical memory differentiation profiles of spike-specific CD8 ${ }^{+} \mathrm{T}$ cells with representative gating and dot plots showing the distribution of subsets across individuals with detectable responses. f, Functional profiles of spike-specific CD8 ${ }^{+}$T cells in BNT162b2-vaccinated individuals with representative gating and pie charts showing the mean frequency for each combination. Polyfunctional responses were compared using a permutation test. Data in bar charts are shown as mean $\pm 95 \%$ confidence intervals, and data in dot plots are shown as median \pm interquartile range. Each dot represents one donor. GrzB, granzyme B; NS, not significant.

\section{Reference}

1. Dyer, O. COVID-19: Omicron is causing more infections but fewer hospital admissions than delta, South African data show. BMJ 375, n3104 (2021).

2. Cameroni, E. et al. Broadly neutralizing antibodies overcome SARS-CoV-2 Omicron antigenic shift. Nature https://doi.org/10.1038/s41586-021-04386-2 (2021).
3. Liu L. et al. Striking antibody evasion manifested by the Omicron variant of SARS-CoV-2. Nature https://doi.org/10.1038/s41586-021-04388-0 (2021).

4. Collie, S., Champion, J., Moultrie, H., Bekker, L. G. \& Gray, G. Effectiveness of BNT162b2 vaccine against Omicron variant in South Africa. N. Engl. J. Med. https://doi.org/10.1056/NEJMc2119270 (2021). 
5. Goga, A., et al. Breakthrough COVID-19 infections during periods of circulating Beta, Delta and Omicron variants of concern, among health care workers in the Sisonke Ad26.COV2.S vaccine trial, South Africa. Preprint at medRxiv https://doi.org/10.1101/2021.12.21.21268171 (2021).

6. Ferguson, N., et al.; on behalf of the Imperial College COVID-19 response team. Report 50: Hospitalisation Risk for Omicron Cases in England. (Imperial College London, 2021)

7. Wolter, N. et al. Early assessment of the clinical severity of the SARS-CoV-2 Omicron variant in South Africa. Lancet 399, 437-446 (2022).

8. Braun, J. et al. SARS-CoV-2-reactive T cells in healthy donors and patients with COVID-19. Nature 587, 270-274 (2020).

9. Grifoni, A. et al. Targets of T cell responses to SARS-CoV-2 coronavirus in humans with COVID-19 disease and unexposed individuals. Cell 181, 1489-1501 (2020).

10. Kalimuddin, S. et al. Early T cell and binding antibody responses are associated with COVID-19 RNA vaccine efficacy onset. Med (N. Y) 2 682-688 (2021).

11. Le Bert, N. et al. SARS-CoV-2-specific T cell immunity in cases of COVID-19 and SARS, and uninfected controls. Nature 584, 457-462 (2020).

12. Oberhardt, V. et al. Rapid and stable mobilization of $\mathrm{CD}^{+} \mathrm{T}$ cells by SARS-CoV-2 mRNA vaccine. Nature 597, 268-273 (2021).

13. Peng, $\mathrm{Y}$. et al. Broad and strong memory $\mathrm{CD} 4^{+}$and $\mathrm{CD} 8^{+} \mathrm{T}$ cells induced by SARS-CoV-2 in UK convalescent individuals following COVID-19. Nat. Immunol. 21, 1336-1345 (2020).

14. Sekine, T. et al. Robust $\mathrm{T}$ cell immunity in convalescent individuals with asymptomatic or mild COVID-19. Cell 183, 158-168 e114 (2020).

15. Israelow, B. et al. Adaptive immune determinants of viral clearance and protection in mouse models of SARS-CoV-2. Sci. Immunol. 6, eabl4509 (2021).

16. McMahan, K. et al. Correlates of protection against SARS-CoV-2 in rhesus macaques. Nature 590, 630-634 (2021).
17. Rydyznski Moderbacher, C. et al. Antigen-specific adaptive immunity to SARS-CoV-2 in acute COVID-19 and associations with age and disease severity. Cell 183, 996-1012 (2020).

18. Tan, A. T. et al. Early induction of functional SARS-CoV-2-specific T cells associates with rapid viral clearance and mild disease in COVID-19 patients. Cell Rep. 34, 108728 (2021).

19. Tarke, A. et al. Impact of SARS-CoV-2 variants on the total CD4 ${ }^{+}$and CD8 ${ }^{+}$ $\mathrm{T}$ cell reactivity in infected or vaccinated individuals. Cell Rep. Med. 2, 100355 (2021).

20. Meng B., et al. SARS-CoV-2 Omicron spike mediated immune escape, infectivity and cell-cell fusion. Preprint at bioRxiv https://doi. org/10.1101/2021.12.17.473248 (2021).

21. Niessl, J. et al. Identification of resident memory $\mathrm{CD}^{+} \mathrm{T}$ cells with functiona specificity for SARS-CoV-2 in unexposed oropharyngeal lymphoid tissue. Sci. Immunol. 6, eabk0894 (2021).

Publisher's note Springer Nature remains neutral with regard to jurisdictional claims in published maps and institutional affiliations.

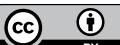

Open Access This article is licensed under a Creative Commons Attribution 4.0 International License, which permits use, sharing, adaptation, distribution and reproduction in any medium or format, as long as you give appropriate credit to the original author(s) and the source, provide a link to the Creative Commons license, and indicate if changes were made. The images or other third party material in this article are included in the article's Creative Commons license, unless indicated otherwise in a credit line to the material. If material is not included in the article's Creative Commons license and your intended use is not permitted by statutory regulation or exceeds the permitted use, you will need to obtain permission directly from the copyright holder. To view a copy of this license, visit http://creativecommons. org/licenses/by/4.0/.

(c) The Author(s) 2022 


\section{Methods}

Samples. Healthy individual volunteers $(n=40)$ were sampled 6 months after a second dose of the BNT162b2 vaccine (Pfizer/BioNTech) as part of a clinical trial registered at EudraCT (2021-000175-37) (ref. ${ }^{22}$ ). Two standard doses of the vaccine were administered with an interval of 21 days. The study was approved by the Swedish Medical Product Agency (ID 5.1-2021-5881). Additional samples ( $n=15$ ) were collected 3 months after the second dose for validation purposes (Extended Data Fig. 1). Convalescent individual volunteers were sampled 9 months after reverse-transcription polymerase chain reaction-verified infection with SARS-CoV-2 leading to mild (nonhospitalized, $n=26$ ) or severe (hospitalized, $n$ $=22$ ) disease during the first wave of the pandemic in March-April 2020, before the emergence of the Alpha, Beta and Delta VOCs. None of these individuals had received a COVID-19 vaccine at the time of sample collection. Seronegative volunteer samples were acquired from healthy blood donors in late 2020. The absence of spike-specific antibodies was confirmed using the Anti-SARS-CoV-2 S Immunoassay (Roche). Cohort details are summarized in Supplementary Table 1. All participants provided written informed consent in accordance with the principles of the Declaration of Helsinki. Convalescent and seronegative cohorts were approved by the Regional Ethics Committee in Stockholm, Sweden. Population characteristics of each cohort were not considered and did not factor in for inclusion into this study. PBMCs were isolated via standard density gradient centrifugation and cryopreserved in fetal bovine serum (FBS) containing $10 \%$ dimethyl sulfoxide (DMSO).

Peptides. Overlapping peptides were designed to span the entire spike protein sequence of SARS-CoV-2 corresponding to the ancestral Wuhan strain (wild-type) or B.1.1.529. Test peptides comprising $15 \mathrm{mers}$ overlapping by ten amino acids were synthesized as crude material for functional screens (TC Peptide Lab). Validation peptides comprising 20 mers overlapping by ten amino acids were synthesized to an equivalent specification (Sigma-Aldrich). All peptides were reconstituted in DMSO, diluted to stock concentrations of $100 \mu \mathrm{g} \mathrm{ml}^{-1}$ in phosphate-buffered saline (PBS) and stored at $-20^{\circ} \mathrm{C}$.

Activation-induced marker assays. PBMCs were thawed quickly; resuspended in RPMI 1640 containing $10 \%$ FBS, $1 \%$ L-glutamine and $1 \%$ penicillin/streptomycin (complete medium) in the presence of DNase I $\left(10 \mathrm{U} \mathrm{ml}^{-1}\right.$, Sigma-Aldrich); and rested at $1 \times 10^{6}$ cells per well in 96-well U-bottom plates (Corning) for 4 $\mathrm{h}$ at $37^{\circ} \mathrm{C}$. The medium was then supplemented with anti-CXCR5-BB515 and anti-CD40 (unconjugated), followed 15 min later by the relevant peptide pools ( 1 $\mu \mathrm{g} \mathrm{ml}^{-1}$ per peptide). Negative control wells contained equivalent DMSO. After 12 $\mathrm{h}$, cells were washed in PBS supplemented with $2 \%$ FBS and $2 \mathrm{mM}$ EDTA (FACS buffer) and stained with anti-CCR4/CD194-BB700, anti-CCR6/CD196-BUV737, anti-CCR7-APC-Cy7 and anti-CXCR3-AF647 for $10 \mathrm{~min}$ at $37^{\circ} \mathrm{C}$. Additional surface stains were performed for $30 \mathrm{~min}$ at room temperature in the presence of Brilliant Stain Buffer Plus (BD Biosciences). Viable cells were identified by exclusion using a LIVE/DEAD Fixable Aqua Dead Cell Stain Kit (Thermo Fisher Scientific). Stained cells were washed in FACS buffer, fixed in PBS containing $1 \%$ paraformaldehyde (Biotium), and acquired using a FACSymphony A5 (BD Biosciences). The gating strategy is shown in Extended Data Fig. 1. All flow cytometry reagents are detailed in Supplementary Table 2.

Intracellular cytokine staining. PBMCs were thawed quickly, resuspended in complete medium in the presence of DNase $\mathrm{I}\left(10 \mathrm{U} \mathrm{ml}^{-1}\right.$, Sigma-Aldrich $)$ and rested at $1 \times 10^{6}$ cells per well in 96-well U-bottom plates (Corning) for $4 \mathrm{~h}$ at $37^{\circ} \mathrm{C}$. The medium was then supplemented with anti-CXCR5-BB515, followed $15 \mathrm{~min}$ later by the relevant peptide pools $\left(1 \mu \mathrm{g} \mathrm{m}{ }^{-1}\right.$ per peptide $)$ and a further $1 \mathrm{~h}$ later by brefeldin A ( $1 \mu \mathrm{g} \mathrm{ml}^{-1}$, Sigma-Aldrich), monensin $\left(0.7 \mu \mathrm{g} \mathrm{ml}^{-1}, \mathrm{BD}\right.$ Biosciences) and anti-CD107a-BV785. Negative control wells contained equivalent DMSO. After $9 \mathrm{~h}$, cells were washed in FACS buffer and stained with anti-CCR4/ CD194-BB700, anti-CCR6/CD196-BUV737, anti-CCR7-APC-Cy7 and anti-CXCR3-BV750 for $10 \mathrm{~min}$ at $37^{\circ} \mathrm{C}$. Additional surface stains were performed for $30 \mathrm{~min}$ at room temperature in the presence of Brilliant Stain Buffer Plus (BD Biosciences). Viable cells were identified by exclusion using a LIVE/DEAD Fixable Aqua Dead Cell Stain Kit (Thermo Fisher Scientific). Cells were then washed in FACS buffer and fixed/permeabilized using a FoxP3 Transcription Factor Staining Buffer Set (Thermo Fisher Scientific). Intracellular stains were performed for 30 min at room temperature. Stained cells were washed in FACS buffer, fixed in PBS containing $1 \%$ paraformaldehyde (Biotium) and acquired using a FACSymphony A5 (BD Biosciences). All flow cytometry reagents are detailed in Supplementary Table 2.

Data analysis and statistics. All samples from each cohort were randomly assigned and analyzed with wild-type and Omicron variant peptides in the same experiment. Flow cytometry data were analyzed using FlowJo version 10.8.0 (FlowJo). Stimulation indices were calculated as fold change in frequency relative to the negative control (equivalent DMSO). Positive responses were identified using a threshold stimulation index $>2$ to exclude background or nonspecific responses. Only memory populations were included for the analysis of spike-specific responses by the exclusion of the naive subset $\left(\mathrm{CD} 45 \mathrm{RA}{ }^{+} \mathrm{CCR} 7^{+}\right)$.
Data exclusion criteria were established before all experiments. The investigators were not blinded to allocation during experiments and outcome assessment. Statistical analyses were performed using Prism version 9 (GraphPad). Significance between paired groups was assessed using two-sided Wilcoxon signed rank tests. Functional profiles were deconvoluted using Boolean gating in FlowJo version 10.8.0 (FlowJo) followed by downstream analyses in SPICE version 6.1 (https:// niaid.github.io/spice/).

Reporting Summary. Further information on research design is available in the Nature Research Reporting Summary linked to this article.

\section{Data availability}

All requests for raw and analyzed preclinical data and materials will be promptly reviewed by the corresponding author (M.B.) to determine if they are subject to intellectual property or confidentiality obligations. Any data and materials that can be shared will be released via a material transfer agreement (requested to M.B.). Personal data underlying this article cannot be shared publicly as they are sensitive. Enquiries regarding data availability should be directed to marcus.buggert@ki.se.

\section{References}

22. Bergman, P. et al. Safety and efficacy of the mRNA BNT162b2 vaccine against SARS-CoV-2 in five groups of immunocompromised patients and healthy controls in a prospective open-label clinical trial. EBioMedicine 74, 103705 (2021).

\section{Acknowledgements}

This project was supported by grants from the SciLifeLab National COVID-19 Research Program, financed by the Knut and Alice Wallenberg Foundation, the Swedish Research Council, Nordstjernan AB, Region Stockholm and the Karolinska Institutet. J.N. was supported by an EMBO postdoctoral fellowship (ALTF 1062-2020). D.A.P. was supported by the National Institute for Health Research (COV-LT2-0041). A.C.K. was supported by the Swedish Research Council (2020-02033), the Centrum för Innovativ Medicin (20190495) and the Karolinska Insitutet (2019-00931). M.B. was supported by the Swedish Research Council, the Knut and Alice Wallenberg Foundation, the Karolinska Institutet, the Swedish Society of Medicine, the Swedish Cancer Society, the Swedish Childhood Cancer Fund, the Åke Wibergs Stiftelse and the Jonas Söderquist Stiftelse. This project was also supported in part by federal funds from the National Institute of Allergy and Infectious Diseases, National Institutes of Health, Department of Health and Human Services under contracts 75N93021C00016 (A.S.) and 75N9301900065 (D.W. and A.S.). We thank the COVAXID clinical study group, P. Nowak, E. Smith, P. Ljungman, S. Mielke, G. Söderdahl and O. Blennow, for their involvement in the COVAXID study and recruitment of vaccinated healthy study subjects.

\section{Author contributions}

Conceptualization, Y.G., C.C., J.K.S., D.A.P., H.-G.L., A.C.K., A.S., S.A. and M.B. Sample collection, Y.G., C.C., T.R.M., J.N., L.H., A. Österborg, P.B., P.C., A. Olsson, H.-G.L., S.A. and M.B. Investigation, Y.G., C.C., A.G., A. Olofsson, M.H. and M.B. Formal analysis, Y.G. and C.C. Visualization, Y.G., C.C. and M.B. Resources, A.G., J.K.S., D.W., H.-G.L, A.C.K., A.S., S.A. and M.B. Funding acquisition, D.A.P., H.-G.L., A.S., S.A. and M.B. Supervision, J.K.S., H.-G.L., S.A. and M.B. Writing - original draft, Y.G., C.C., D.A.P. and M.B. Writing - review \& editing, Y.G., C.C., A.G., T.R.M., J.N., A. Österborg, P.B., J.K.S., D.A.P., H.-G.L., A.C.K., S.A. and M.B.

\section{Funding}

Open access funding provided by Karolinska Institute.

\section{Competing interests}

A.S. is a consultant for Gritstone Bio, Flow Pharma, Arcturus Therapeutics, ImmunoScape, CellCarta, Avalia, Moderna, Fortress and Repertoire, and the La Jolla Institute has filed patents to protect various aspects of the T cell epitope and vaccine design work. S.A. has received honoraria from Gilead, AbbVie, MSD and Biogen and research grants from Gilead and AbbVie. M.B. is a consultant for Oxford Immunotec. The other authors declare no conflicts of interest.

\section{Additional information}

Extended data is available for this paper at https://doi.org/10.1038/s41591-022-01700-x.

Supplementary information The online version contains supplementary material available at https://doi.org/10.1038/s41591-022-01700-x.

Correspondence and requests for materials should be addressed to Marcus Buggert.

Peer review information Nature Medicine thanks Antonio Bertoletti and Robert Thimme for their contribution to the peer review of this work. Saheli Sadanand was the primary editor on this article and managed its editorial process and peer review in collaboration with the rest of the editorial team.

Reprints and permissions information is available at www.nature.com/reprints. 


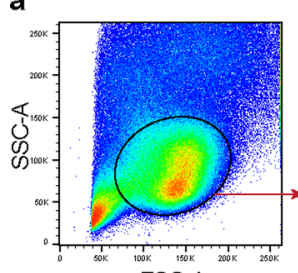

FSC-A

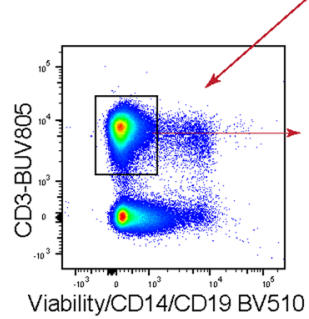

b

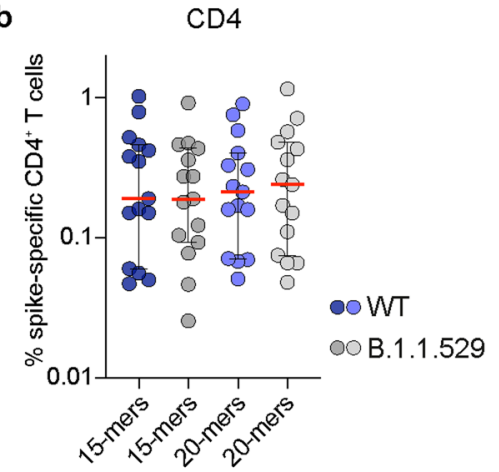

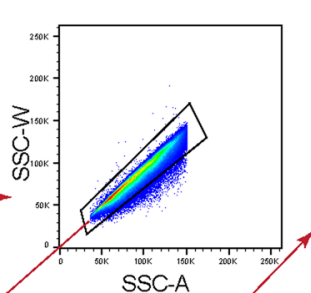
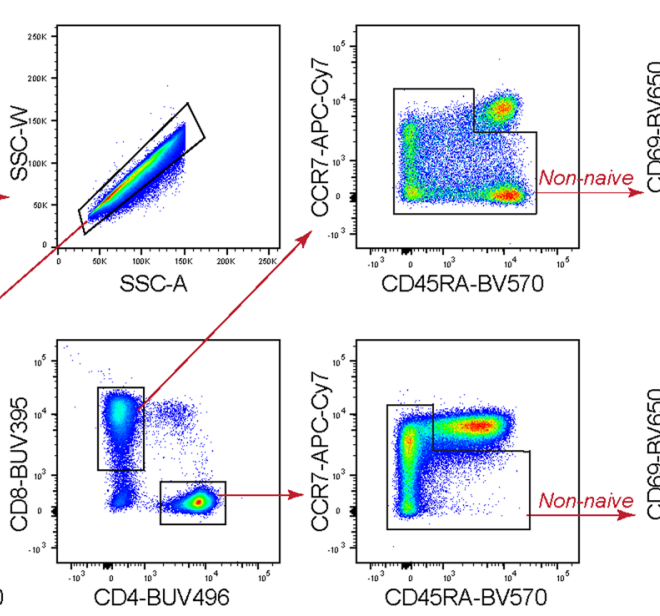

C

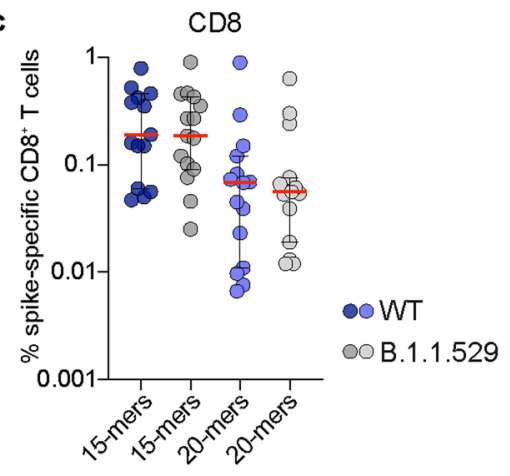

DMSO
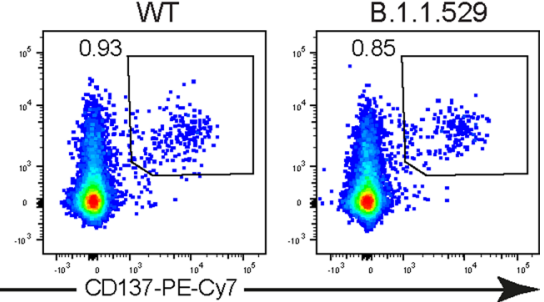

B.1.1.529
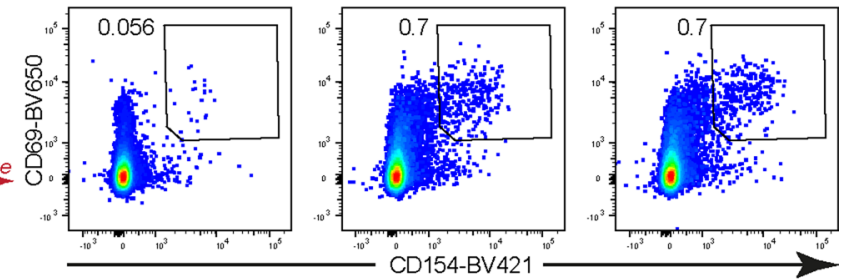

d
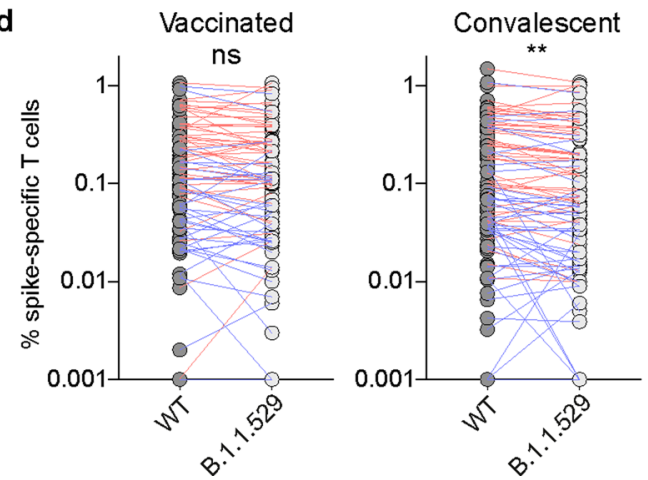

Extended Data Fig. 1 | Peptide validation experiments. a, Representative flow cytometry plots showing the gating strategy used to assess spike-specific $\mathrm{CD}^{+}$and $\mathrm{CD} 8^{+} \mathrm{T}$ cell responses to peptide pools representing wild-type SARS-CoV-2 (WT) or B.1.1.529. b,c, Frequencies of spike-specific CD4 ${ }^{+}$(b) and $\mathrm{CD}^{+} \mathrm{T}$ cells (c) in BNT162b2-vaccinated individuals, comparing test $15 \mathrm{mer}$ peptide pools versus validation 20 mer peptide pools representing wild-type SARS-CoV-2 (WT) or B.1.1.529. d, Pairwise analysis of spike-specific CD4+ (red lines) and CD8 ${ }^{+}$T cell responses (blue lines) in BNT162b2-vaccinated and convalescent individuals. Data in dot plots are shown as median \pm IQR. Each dot represents one donor. ${ }^{\star \star} P=0.007$ (Two-sided Wilcoxon signed rank test). ns, not significant. 


\section{Reporting Summary}

Nature Portfolio wishes to improve the reproducibility of the work that we publish. This form provides structure for consistency and transparency in reporting. For further information on Nature Portfolio policies, see our Editorial Policies and the Editorial Policy Checklist.

\section{Statistics}

For all statistical analyses, confirm that the following items are present in the figure legend, table legend, main text, or Methods section.

$\mathrm{n} / \mathrm{a}$ Confirmed

$\bigotimes$ The exact sample size $(n)$ for each experimental group/condition, given as a discrete number and unit of measurement

$\square$ A statement on whether measurements were taken from distinct samples or whether the same sample was measured repeatedly

The statistical test(s) used AND whether they are one- or two-sided

Only common tests should be described solely by name; describe more complex techniques in the Methods section.

Х A description of all covariates tested

Х $\square$ A description of any assumptions or corrections, such as tests of normality and adjustment for multiple comparisons

$\triangle$ A full description of the statistical parameters including central tendency (e.g. means) or other basic estimates (e.g. regression coefficient) AND variation (e.g. standard deviation) or associated estimates of uncertainty (e.g. confidence intervals)

For null hypothesis testing, the test statistic (e.g. $F, t, r$ ) with confidence intervals, effect sizes, degrees of freedom and $P$ value noted Give $P$ values as exact values whenever suitable.

Х $\square$ For Bayesian analysis, information on the choice of priors and Markov chain Monte Carlo settings

Х $\square$ For hierarchical and complex designs, identification of the appropriate level for tests and full reporting of outcomes

$\triangle \square$ Estimates of effect sizes (e.g. Cohen's $d$, Pearson's $r$ ), indicating how they were calculated

\section{Our web collection on statistics for biologists contains articles on many of the points above.}

\section{Software and code}

Policy information about availability of computer code

Data collection Flow cytometry data was collected using a BD FACSymphony A5 flow cytometer.

Data analysis Flow cytometry data was analyzed using FlowJo (Version 10.8.0) and GraphPad Prism (Version 9.0.0).

Polyfunctional analysis was performed using SPICE (Version 6) (available at https://niaid.github.io/spice/).

For manuscripts utilizing custom algorithms or software that are central to the research but not yet described in published literature, software must be made available to editors and

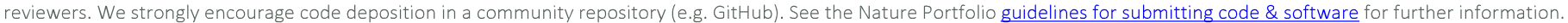

\section{Data}

Policy information about availability of data

All manuscripts must include a data availability statement. This statement should provide the following information, where applicable:

- Accession codes, unique identifiers, or web links for publicly available datasets

- A description of any restrictions on data availability

- For clinical datasets or third party data, please ensure that the statement adheres to our policy

All requests for raw and analyzed preclinical data and materials will be promptly reviewed by the corresponding author (M.B.) to determine if they are subject to intellectual property or confidentiality obligations. Any data and materials that can be shared will be released via a material transfer agreement (requested to M.B.). Personal data underlying this article cannot be shared publicly as they are sensitive. Enquiries regarding data availability should be directed to marcus.buggert@ki.se. 
Please select the one below that is the best fit for your research. If you are not sure, read the appropriate sections before making your selection.

$\bigotimes$ Life sciences $\quad \square$ Behavioural \& social sciences $\square$ Ecological, evolutionary \& environmental sciences

For a reference copy of the document with all sections, see nature.com/documents/nr-reporting-summary-flat.pdf

\section{Life sciences study design}

All studies must disclose on these points even when the disclosure is negative.

Sample size With a sample size of $n=20$ per group, the probability is $80 \%$ that the study will detect a relationship between the independent and the dependent variables at a two-sided 0.05 significance level, if the true change in the dependent variables is 0.663 standard deviations per one standard deviation change in the independent variable. Based on previous experience (Sekine et al, 2020, Cell etc), it should therefore be possible to detect group differences.

The total number of individuals from each cohort were selected to match as closely as possible and be approximately twice as high as our power analysis. Vaccinated $n=40$. Convalescent $n=48$. Seronegative $n=48$.

Data exclusions Individuals with a stimulation index less than 2 were excluded from downstream phenotypic and functional analyses to minimise analysis of background or non-specific responses. Only memory populations were included for the analysis of spike-specific responses by the exclusion of the naive subset (CD45RA+CCR7+). Data exclusion criteria were established before all experiments, similar to before Niessl et al, 2020, Science Immunol etc).

Replication Given limited sample availability, replication was not performed.

Randomization Individuals were randomly analyzed. However, WT and Omicron peptides were supplemented in the same experiments to avoid intraindividual experimental differences of $T$ cell responses against the different viral variants.

Blinding Investigators were not blinded. The data generation for all samples within the same cohort were run in parallel in single experiments.

\section{Reporting for specific materials, systems and methods}

We require information from authors about some types of materials, experimental systems and methods used in many studies. Here, indicate whether each material, system or method listed is relevant to your study. If you are not sure if a list item applies to your research, read the appropriate section before selecting a response.

\begin{tabular}{l|l} 
Materials \& experimental syste \\
\hline $\mathrm{n} / \mathrm{a}$ & Involved in the study \\
\hline & $\bigotimes$ Antibodies \\
\hline & $\square$ Eukaryotic cell lines \\
\hline & $\square$ Palaeontology and archaeology \\
$\square$ & $\square$ Clinical data \\
$\searrow$ & $\square$ Dual use research of concern
\end{tabular}

Methods $\mathrm{n} / \mathrm{a}$ Involved in the study

$\triangle \square$ ChIP-seq

$\square$ \ Flow cytometry

Х| MRI-based neuroimaging

\section{Antibodies}

Antibodies used

\author{
AlM assay: \\ Fixable Aqua Viability dye Thermo Fisher Cat\#L34957 Dilution 3:5000 \\ BUV805 CD3 BD Biosciences Clone UCHT1 Cat\#612895 Dilution 1:50 \\ BUV496 CD4 BD Biosciences Clone SK3 Cat\#612936 Dilution 1:25 \\ BUV395 CD8 BD Biosciences Clone RPA-T8 Cat\#563795 Dilution 1:250 \\ BV510 CD14 BioLegend Clone M5E2 Cat\#301842 Dilution 1:100 \\ BV510 CD19 BioLegend Clone HIB19 Cat\#302242 Dilution 1:100 \\ BV570 CD45RA BioLegend Clone HI100 Cat\#304132 Dilution 1:200 \\ APC-Cy7 CCR7 BioLegend Clone G043H7 Cat\#353212 Dilution 1:50 \\ PE-CY7 CD137 BioLegend Clone 4B4-1 Cat\#309818 Dilution 1:25 \\ BV421 CD154 BioLegend Clone 24-31 Cat\#310824 Dilution 1:25 \\ BB700 CD194 BD Biosciences Clone 1G1 Cat\#566475 Dilution 1:50 \\ BUV737 CD196 BD Biosciences Clone 11A9 Cat\#612780 Dilution 1:500 \\ BB515 CXCR5 BD Biosciences Clone RF8B2 Cat\#564624 Dilution 1:100 \\ Unconjugated CD40 Miltenyi Biotec Clone HB14 Cat\#130-094-133 Dilution 1:200 \\ BV650 CD69 BioLegend Clone FN50 Cat\#310934 Dilution 1:50
}


AF647 CXCR3 BioLegend Clone G025H7 Cat\#353712 Dilution 1:200

Intracellular staining:

Fixable Aqua Viability dye Thermo Fisher Cat\#L34957 Dilution 3:5000

BUV805 CD3 BD Biosciences Clone UCHT1 Cat\#612895 Dilution 1:250

BUV496 CD4 BD Biosciences Clone SK3 Cat\#612936 Dilution 1:25

BUV395 CD8 BD Biosciences Clone RPA-T8 Cat\#563795 Dilution 1:250

BV510 CD14 BioLegend Clone M5E2 Cat\#301842 Dilution 1:100

BV510 CD19 BioLegend Clone HIB19 Cat\#302242 Dilution 1:100

BV570 CD45RA BioLegend Clone HI100 Cat\#304132 Dilution 1:200

APC-Cy7 CCR7 BioLegend Clone G043H7 Cat\#353212 Dilution 1:50

PE-Cy7 CD137 BioLegend Clone 4B4-1 Cat\#309818 Dilution 1:100

BV421 CD154 BioLegend Clone 24-31 Cat\#310824 Dilution 1:25

BB700 CD194 BD Biosciences Clone 1G1 Cat\#566475 Dilution 1:50

BUV737 CD196 BD Biosciences Clone 11A9 Cat\#612780 Dilution 1:500

BB515 CXCR5 BD Biosciences Clone RF8B2 Cat\#564624 Dilution 1:100

BUV563 CD69 BD Biosciences Clone FN50 Cat\#748764 Dilution 1:200

BV785 CD107a BioLegend Clone H4A3 Cat\#328644 Dilution 1:500

BV750 CXCR3 BD Biosciences Clone 1C6 Cat\#746894 Dilution 1:50

BB790 Granzyme B BD Biosciences Clone GB11 Cat\#624296 Dilution 1:500

PE IFN- $\gamma$ BioLegend Clone B27 Cat\#506507 Dilution 1:400

PE-Dazzle594 IL-2 BioLegend Clone MQ1-17H12 Cat\#500344 Dilution 3:100

BV711 PD1 BioLegend Clone EH12.2H7 Cat\#329928 Dilution 1:25

BV650 TNF BD Biosciences Clone MAb11 Cat\#563418 Dilution 3:500

Validation

All antibodies are validated by their respective manufacturers and are quality control tested by surface or intracellular immunofluorescent staining with flow cytometric analysis. For more information on the antibodies used, please visit bioledend.com,

bdbiosciences.com, thermofisher.com and miltenyibiotec.com.

\section{Human research participants}

\section{Policy information about studies involving human research participants}

Population characteristics

Recruitment

Ethics oversight
Among the 40 vaccinated individuals, all were adults with an age range of 22-79 (median age of 53), with females comprising $58 \%$. All 48 convalescent patients confirmed by positive RT-PCR results for SARS-CoV-2 fell within the age range of 44-68 years (median age of 56), with $23 \%$ of them being females. Personal information of all 48 individuals with seronegative for SARS-COVID-2 is not available. Population characteristics of each cohort were not considered and did not factor in for inclusion into this study.

The vaccinated healthy individuals were recruited between Feb-March 2021 at Karolinska University Hospital, Sweden in a clinical trial (EudraCT no. 2021-000175-37). Most of the healthy individuals were family members of the study participants with immunocompromised disorders (not included in the present study), recruited to the trial for COVID-19 vaccination. Inclusion criteria for healthy individuals were individual $\geq 18$ years, no known immunosuppressive disease or treatment with significant co-morbidity according to the investigator's judgement. The exclusion criteria were previous or ongoing COVID-19, presence of coagulation disease, planned to receive other vaccine within 14 days prior to the first dose of the study vaccine or receive other vaccine from the time of the first study vaccine dose until 14 days after the second dose of study vaccine, pregnancy or breast-feeding, hypersensitivity to any of the active substance in the vaccine, cannot comprehend the information given to study participants for consent, or individuals of any other reasons judged by the investigator to be not suitable for inclusion in the study. One potential selection bias could be the participants willingness for COVID-19 vaccination and serial venipuncture performed in this study, but it is unlikely that this could have impacted any of the results in this study significantly.

The convalescent individuals have been tested positive for SARS-CoV-2 infection during March-April 2020 at Karolinska University Hospital. Patients with severe COVID-19 have been hospitalized, while those with mild COVID-19 have been followed-up at the outpatient clinic of the department of Infectious Diseases, Karolinska University Hospital. The patients were recruited while in the convalescence phase, with blood samples in this study collected at 9 months after the verified SARS-CoV-2 infection. One potential selection bias could be the participants willingness for serial venipuncture or other features associated with individuals who declined enrollment into our study, but it is unlikely that this could have impacted any of the results significantly.

Seronegative samples were acquired from healthy blood donors in late 2020.

All study participants provided written informed consent. All study participants were volunteers.

The vaccinated cohort was approved by the Swedish Medical Product Agency (ID 5.1-2021-5881) and the Swedish Ethical Review Authority (ID 2021-00451). Other cohorts were approved by the Regional Ethics Committee in Stockholm, Sweden. 
Plots

Confirm that:

\The axis labels state the marker and fluorochrome used (e.g. CD4-FITC).

\The axis scales are clearly visible. Include numbers along axes only for bottom left plot of group (a 'group' is an analysis of identical markers).

\All plots are contour plots with outliers or pseudocolor plots.

$\bigotimes$ A numerical value for number of cells or percentage (with statistics) is provided.

\section{Methodology}

Sample preparation

Instrument

Software

Cell population abundance

Gating strategy

$\bigotimes$ Tick this box to confirm that a figure exemplifying the gating strategy is provided in the Supplementary Information.
Cryopreserved PBMC

BD FACSymphony A5

FlowJo version 10.8 .0

No sorting was performed.

Gating strategies are shown throughout the figures. Briefly, lymphocytes were gated by standard FSC/SSC gating followed by singlet discrimination. Viability staining was used to gate live cells. T cells were gated by CD3 expression and no expression of the lineage markers CD19 and CD14. CD4 and CD8 T cells were identified by CD4 and CD8 staining. Naive cells with CD45RA and CCR7 high expression were gated out. Within the remaining population, spike-specific CD4 and CD8 responses were identified by coexpression of CD69/CD154 or CD69/CD137 respectively. 